\title{
A novel UHF radar system design for river dynamics monitoring
}

\author{
Ke Li ${ }^{1 \mathrm{a})}$, Biyang Wen ${ }^{1 b)}$, Yamin $\mathrm{Xu}^{1}, \operatorname{Jian}_{\mathrm{Tan}^{1}}{ }^{1}$ \\ Jing Yang ${ }^{1}$, and Yu Liu ${ }^{2}$ \\ ${ }^{1}$ Electronic Information School, Wuhan University, \\ Wuhan, Hubei Province, 430072, China \\ ${ }^{2}$ Xiantao Hydrological Station, Yangtze River Water Resource Commission \\ of the Ministry of Water Resource, Xiantao, Hubei Province, 430000, China \\ a)like_@whu.edu.cn \\ b)rspl@whu.edu.cn
}

\begin{abstract}
This paper proposes a novel ultra high frequency (UHF) radar system for river dynamics parameters monitoring. As compared with conventional radar system: (1) Intermediate frequency (IF) sampling mode of traditional superheterodyne receivers is replaced by the direct radio frequency (RF) band-pass sampling and orthogonal demodulation (2) Multichannel antenna system is divided into two units, which realizes that one radar system operation obtains two-dimensional current vectors. Results of field test compared with EKZ-I current measurement at Xiantao Hydrological Station, China from August to September in 2014 are given to demonstrate the correctness and effectiveness of this system.
\end{abstract}

Keywords: UHF radar, direct RF sampling, two-dimensional current vector, river monitoring

Classification: Electron devices, circuits, and systems

\section{References}

[1] C. C. Teague, D. E. Barrick, P. M. Lilleboe and R. T. Cheng: IGRASS (2001) 1288. DOI:10.1109/IGARSS.2001.976821

[2] C. C. Teague: IGARSS (2008) 890. DOI:10.1109/IGARSS.2008.4779866

[3] D. E. Barrick, C. C. Teague and P. M. Lilleboe: U.S Patent 7688251 (2008).

[4] C. M. Keller, J. M. Burkhart and T. T. Phuong: ICUWB (2007) 387. DOI:10. 1109/ICUWB.2007.4380975

[5] Z. S. Yan, B. Y. Wen and C. J. Wang: IEICE Electron. Express 6 (2009) 780. DOI:10.1587/elex.6.780

[6] D. D. Crombie: Nature 175 (1955) 681. DOI:10.1038/175681a0

[7] E. B. Joffe and K. S. Lock: GROUNDS FOR GROUNDING A Circuit-toSystem Handbook (Wiley, New Jersey, 2010) 589.

[8] D. E. Barrick, B. J. Lipa and P. M. Lilleboe: U.S Patent 5361072 (1992).

[9] Y. M. Xu, B. Y. Wen and K. Li: J. Huazhong Univ. of Sci. \& Tech. 42 (2014) 64. DOI:10.13245/j.hust.140914

[10] J. Leise: IEEE J. Oceanic Eng. 9 (1984) 106. DOI:10.1109/JOE.1984.1145604 


\section{Introduction}

In the last decade, the non-contact method of measuring has become a popular way to realize hourly mapping of river current, such as the product called RiverSonde radar system with superheterodyne structure of CODAR company, which has achieved some results in field experiments $[1,2,3]$. However, in order to obtain two-dimensional current vectors, two or more radar systems have to be used simultaneously in normal operation, with highly strict clock synchronization among these remote radar sites, thus the multi-station radar system is always labeled by large size and expensive cost.

In this paper, a novel UHF radar system for river dynamics monitoring is presented. Comparing with the traditional radar system: this novel radar system also utilizes the Doppler shift by Bragg scattering of radio waves to detect the river surface velocities and volume flow, whereas upgrades the sampling mode from superheterodyne to direct sampling architecture [4], thus there is nearly no analog circuit in this radar system, the demodulation and pulse compression processes are all accomplished in digital domain. Besides, the receiving antenna system is divided into two units, which realizes the two-dimensional current vectors mapping in one radar system, high cost of multi-station method and synchronization problem in hardware structure are eliminated. In addition to this new structure, the power of transmitting signal is $1 \mathrm{~W}$, high power transmitter is unnecessary in this system.

The structure of this paper is as follows. In section 2, we put forward the hardware composition of the novel radar system and the corresponding waveform parameter design. In Section 3, the detailed realizations of the radar receiver are presented. Signal processing of this novel system is shown in Section 4. The field experiment at Hanjiang river is given in Section 5. Section 6 contains brief conclusions.

\section{Hardware composition and waveform parameters design}

\subsection{Hardware composition}

As demonstrated in Fig. 1, this new radar system is composed of receiver system, antenna system, low power transmitter and computer.

For the receiver system, on the one hand, contrasted with the conventional backscatter radar in architecture, this new radar system realizes direct radio frequency sampling, echo signal is mixed with local oscillator generated by field programmable gate array (FPGA) on the digital board, thus analog mixer and local signal generator are not needed, which avoids analog mixer image problem and intermodulation distortion. On the other hand, differing from other direct RF sampling receivers, this novel system implements the coordinate rotation digital compute (CORDIC) algorithm for echo demodulation [5].

For the antenna system, the antennas adopt "one transmitting, six receiving" method, transmitting and receiving antennas are all replaceable Yagis and vertically polarized. The $3 \mathrm{~dB}$ bandwidth of antenna is $45^{\circ}$. The detailed implementation of antenna system is described in Section 5. 


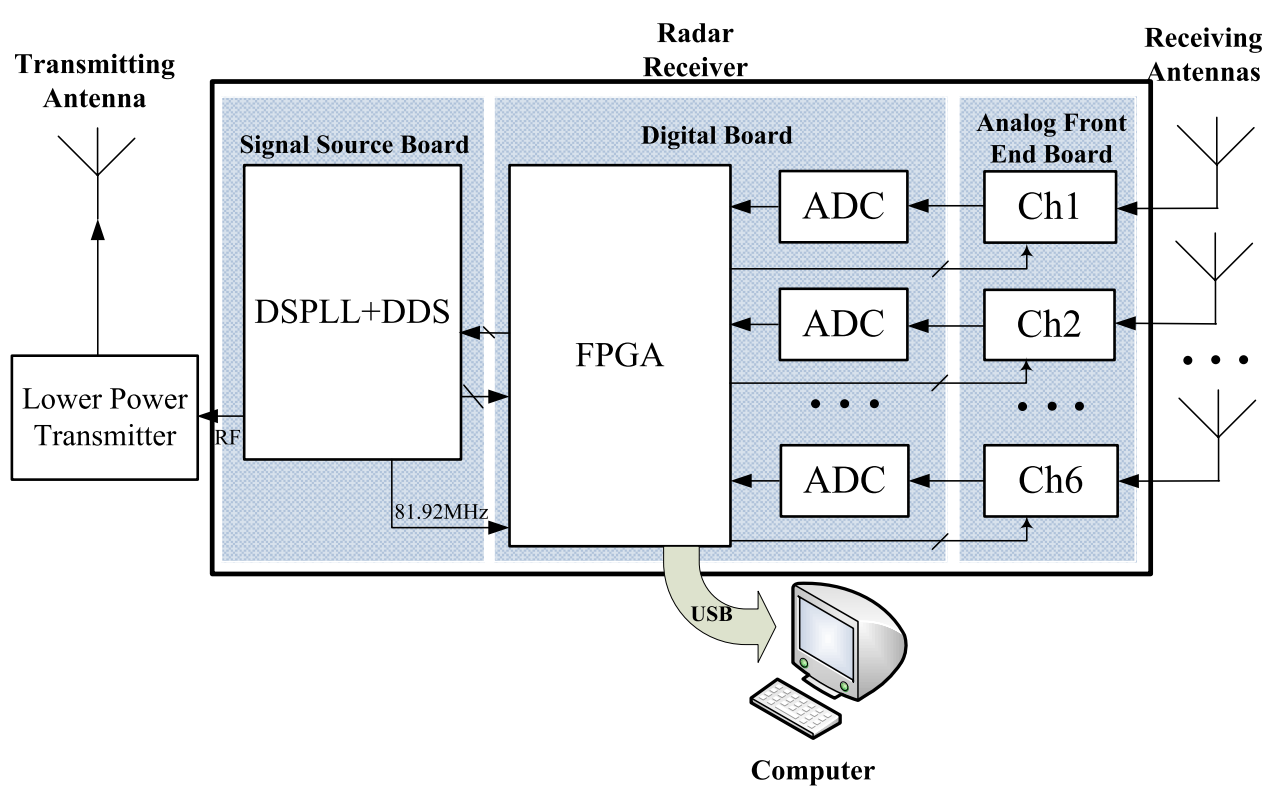

Fig. 1. Architecture of the novel radar system

\subsection{Waveform parameter design}

This radar system adopts the Frequency Modulated Interrupted Continuous Waveform (FMICW) mechanism and operates at $340 \mathrm{MHz}$, which depends on the presence of water waves of $44 \mathrm{~cm}$ wavelength (one-half of the radar wavelength). The sweep bandwidth is $15 \mathrm{MHz}$ and sweep time is $0.04 \mathrm{~s}$, therefore the range resolution is $10 \mathrm{~m}$ and sweep rate of $3.75 \times 10^{8} \mathrm{~Hz} / \mathrm{s}$. The maximum detection range is set to $600 \mathrm{~m}$, which is enough for river monitoring. After orthogonal demodulation, the negative frequency components of baseband are eliminated, therefore the maximal baseband frequency offset is $1.25 \mathrm{KHz}$. The analog-to-digital converter (ADC) sampling frequency of this system is $81.92 \mathrm{MHz}$, there are 640 clock cycles in one pulse repetition interrupt (PRI), so the pulse repetition frequency is $128 \mathrm{KHz}$, which is more than the baseband offset. Besides, based on the theory of Crombie [6], the Bragg-resonant frequency will be $1.88 \mathrm{~Hz}$, the sampling rate of Doppler frequency is $25 \mathrm{~Hz}$, more than twice that of the Bragg frequency too. Consequently, the parameter design will not cause range and Doppler ambiguity.

Due to the short-range river detection, there is no range blind in parameter design of our system. The receiving period will begin as soon as the transmitting period ends in a PRI. In this system, one coherent integration period is consist of 256 sweep periods, resulting in a Doppler resolution of $0.0908 \mathrm{~Hz}$ and velocity resolution of $0.0399 \mathrm{~m} / \mathrm{s}$. According to the waveform parameter design mentioned above, the maximum current speed could be measured is $4.27 \mathrm{~m} / \mathrm{s}$, and the moving target of $5.11 \mathrm{~m} / \mathrm{s}$.

\section{Realization of all module in radar receiver}

As demonstrated above, the radar receiver system consists of Analog Front-End 


\subsection{Analog front-end board}

The AFE board has six isolated but identical structure channels, as shown in Fig. 2, each channel of the AFE board contains two high speed RF switches, three band pass filters, and two wideband amplifiers. The bate ground wave (BGW) signal allows the echo signal derived from the receiving antennas during 3.698 us to 7.812 us in a PRI enter into the AFE. Through amplification and loop filtering, the desired signal is sampled by ADC for further processing. In addition, we can choose the gain control (GC) signal to change the gain of the AFE in different experimental environment. The AFE board achieves $38.4 \mathrm{~dB}$ gain in high gain mode and $20 \mathrm{~dB}$ in low gain mode, sensitivity is $-133 \mathrm{dBm}, 1 \mathrm{~dB}$ compression point $(\mathrm{P} 1 \mathrm{~dB})$ is $-35 \mathrm{dBm}$.

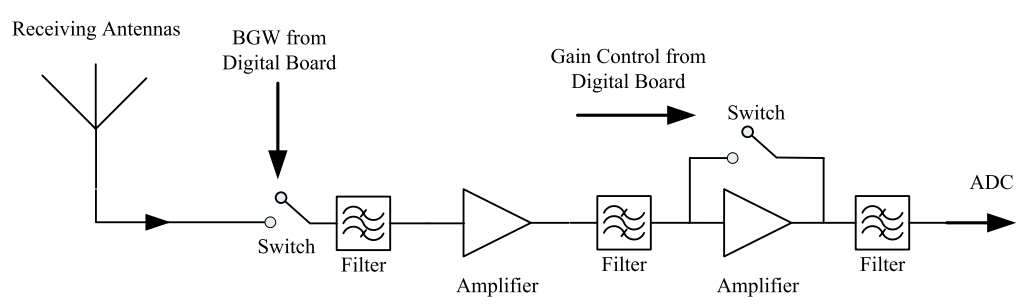

Fig. 2. Architecture of Analog Front-End Board

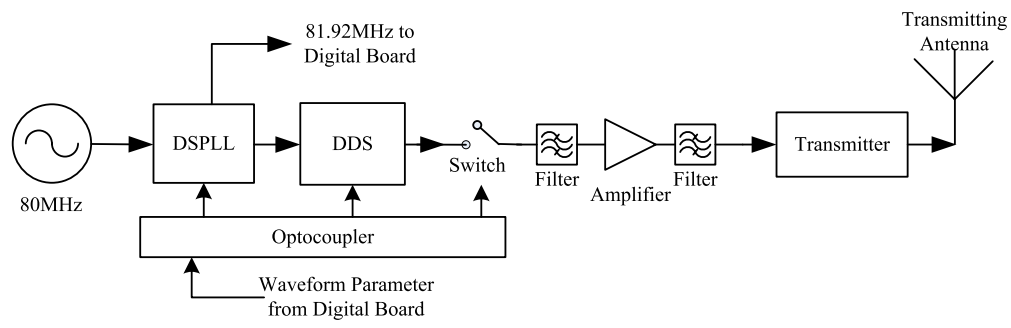

Fig. 3. Architecture of Signal Source Board

\subsection{Signal source board}

This novel radar is a coherent system, thus all clock signals should come from the same clock source. As shown in Fig. 3, a novel model of "DSPLL+DDS" is applied to realize accuracy control of signal phase and frequency under implements of the digital board which offers waveform parameters to the radar system. The $80 \mathrm{MHz}$ clock generated by oven controlled crystal oscillator (OCXO) is multiplied by digital signal phase lock loop (DSPLL), the DSPLL outputs two accurate signals with same initial phase, one is supplied to ADC and FPGA in the digital board with $81.92 \mathrm{MHz}$, the other is $1 \mathrm{GHz}$ to driven direct digital synthesizer (DDS) as its system clock. Then, the DDS outputs center frequency $340 \mathrm{MHz}$ with bandwidth $15 \mathrm{MHz}$ signal, after pre-amplifying and filtering, the signal with $0 \mathrm{dBm}$ is sent to transmitter. The low power transmitter amplifies the $0 \mathrm{dBm}$ signal to $1 \mathrm{~W}$ feeding to the transmitting antenna at last. In order to avoid analog-digital ground interrupt problem [7], the optocoupler is used to keep analog devices, such as DSPLL, DDS and switch from digital device (FPGA). The measurement results indicate that, the 
spur free dynamic range (SFDR) of sweep signal with $15 \mathrm{MHz}$ is better than $63 \mathrm{~dB}$, the phase noise of single frequency level is better than $-101.1 \mathrm{dBc} / \mathrm{Hz} @ 1 \mathrm{KHz}$, the channel flatness is less than $2 \mathrm{~dB}$.

\subsection{Digital board}

As shown in Fig. 4, the digital board is mainly comprised of six ADCs, one FPGA, one clock buffer and one universal serial bus (USB). The clock buffer receives the 81.92 MHz clock from DSPLL and distributes it to FPGA as system clock and to six channel ADCs as sampling clock. The desired signal amplified and filtered by AFE is directly sampled by the ADC with wide analog bandwidth and high sampling rate and then transferred to FPGA, the FPGA utilizes CORDIC algorithm to realize the orthogonal demodulation and cascaded integrator comb (CIC) decimation filter and accomplish high-rate decimation and filtering. The processed data is transmitted to PC by USB with high speed. Finally, we can extract the range and velocity information from the raw data and get a Range-Doppler spectrum displayed on the PC.

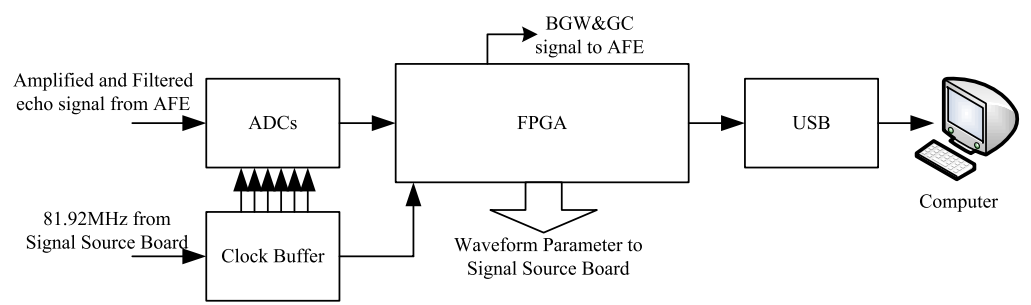

Fig. 4. Architecture of Digital Board

\section{Signal processing}

The raw data is transferred to PC, we could obtain the two-dimensional current vectors after a series of signal processing procedures. The detailed processing chain is shown in Fig. 5.

First of all, the Range-Doppler spectrum is calculated by twice fast fourier transformation (FFT) algorithm [8], the velocity information of certain range bin is

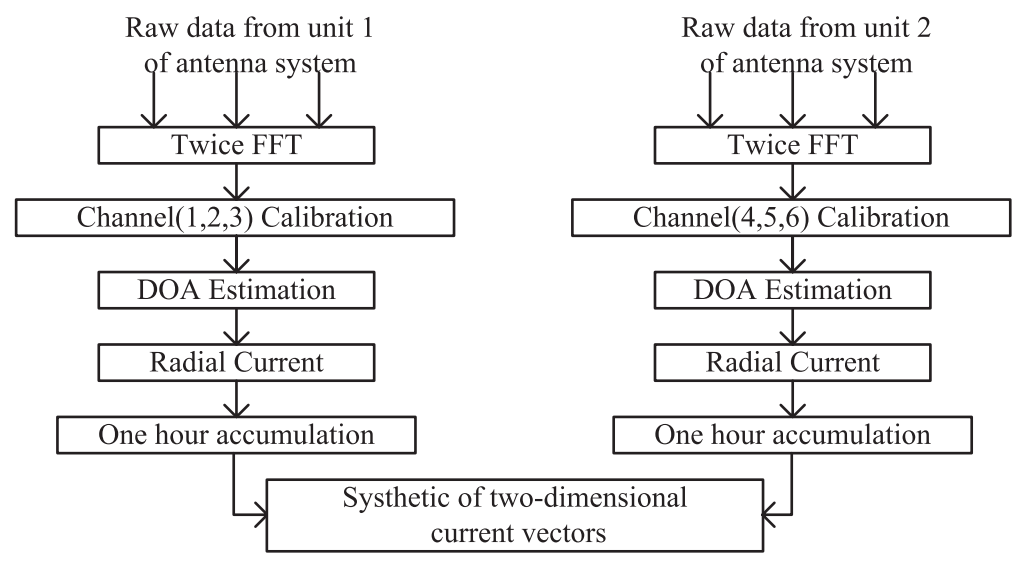

Fig. 5. Signal processing chain 
corresponding to the Doppler frequency. The channel calibration of the two antenna units is accomplished by using the echo signal spectrum of Bragg frequency bin [9]. After that, multiple signal classification (MUSIC) direction finding algorithm realizes the direction of arrival (DOA) estimation of each frequency bin in each range bin and then the radial current map could be generated, after an hour accumulation, the median values of radial current map from two antenna units are applied to synthesize the final two-dimensional current vectors.

\section{Field experiment}

In order to evaluate the performance of this novel radar system in long term observation, an experiment was started on August 6, 2014 and continued through September 9, 2014. The experiment site is on the Hanjiang River, China, which is the biggest tributary of the Yangtze River. The China Yangtze River water resource Commission of the Ministry of water resource implements a river gaging station, the Xiantao Hydrological Station, which provides volume flow data in every 4 hours. Besides, the Xiantao Hydrological Station deployed EKZ-I (a contact current instrument like ADCP) on several occasions during the experiment, to measure horizontal and vertical water flow velocity as compared data with the radar system.

The radar system was installed about $40 \mathrm{~m}$ away from the bank of the Hanjiang River which is about $260 \mathrm{~m}$ wide and $7 \mathrm{~m}$ deep during most of the experiment. It is worth mentioning that, the delay caused by radar receiver was about 6 range bins which was a fixed value of $60 \mathrm{~m}$. Concerning about the distance between the radar system and the river bank, thus we treat the position of $100 \mathrm{~m}$ in Range-Doppler spectrum as the first range bin in the real situation.

The six receiving antennas were divided into two pairs of 3-element array and settled perpendicular to the bank, operated in close proximity with overlapping spatial coverage. The two arrays were about $67 \mathrm{~m}$ apart, the radar system was settled in the middle of these two arrays and connected by low loss cables. A pattern of two-dimensional current vectors was constructed by combining the radial current from the two individual antenna arrays. Fig. 6(a) shows the Doppler spectrum of the 15-th range cell in channel 3. The surface current is measured from the shift of the first-order Bragg peak, as displayed in Fig. 6(a), both of the positive and negative Bragg peaks spread widely due to the richness of the current velocity, the width of which are about $2.0 \mathrm{~Hz}$ (Normalized Doppler Frequency).

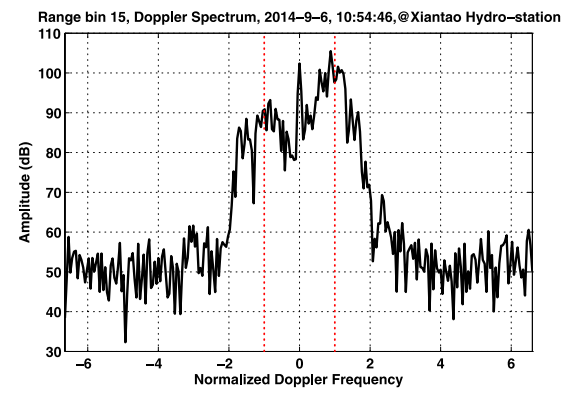

(a) Doppler Spectrum

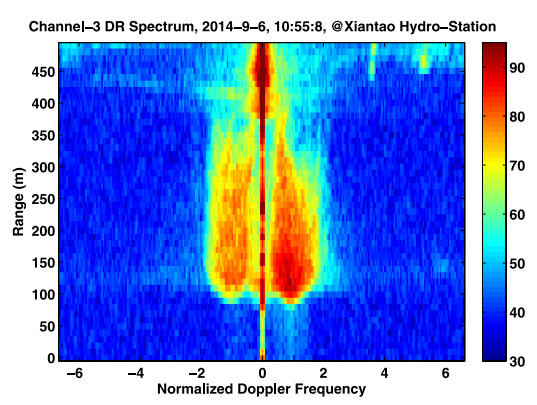

(b) Range-Doppler Spectrum

Fig. 6. Results of Echo Spectrum 
The signal to noise ratio (SNR) of positive Bragg peak exceeds $50 \mathrm{~dB}$, which is enough for further processing to extract current velocities. In the Fig. 6(b), the Bragg peaks cover the entire river, as shown in the Range-Doppler spectrum from $100 \mathrm{~m}$ to nearly $360 \mathrm{~m}$.

The radar data is processed to provide hourly estimates of flow velocity at points separated by $1^{\circ}$ in angle and $10 \mathrm{~m}$ in range over the field of view. A grid of points $10 \mathrm{~m}$ apart is established, and the two-dimensional vectors are computed at each grid point by combining the radial vectors of overlapping coverage. An example of two-dimensional current vectors map, averaged over an hour during a time of relatively calm conditions, is shown in Fig. 7, with the dominant flow from west to east. The echo signals are processed within $\pm 50^{\circ}$ from the perpendicular direction of the antenna system to the bank, and the sharp angular cutoff at the both side of the plot is due to beam width of antennas and signal processing limitations. The figure also shows the banks of channel and the locations of two pairs of antenna (marked out by the red dots). The flow pattern is fairly uniform and parallel to the banks as expected, although there are some instable deviations from the pattern, particularly near the bank. These instabilities are caused by the inherent shortcomings of the radial measurement [10].

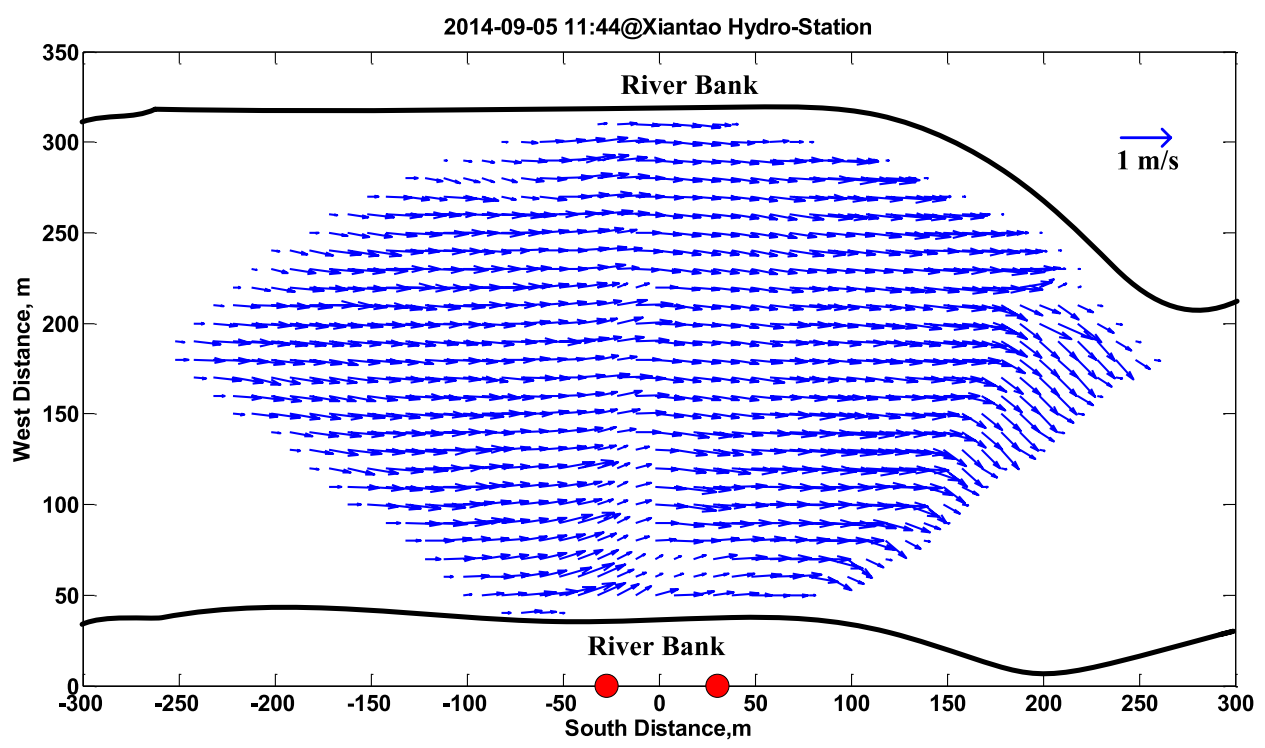

Fig. 7. Two-dimensional current vectors map

Fig. 8(a) demonstrates the radial and vector flow value comparing with EKZ-I before and after combination, agreement between the combined current vectors of radar system and the contact instrument $E K Z-I$ in the same cross-channel profile is good with a correlation coefficient of 0.9878 , which is better than the radial ones ( 0.88 or 0.95 ). The changes of volume from August 9 to 26, 2014 is shown in Fig. 8(b), the volume flow difference between of these two systems is less than $10 \%$ when using the same bottom profile of the river. 


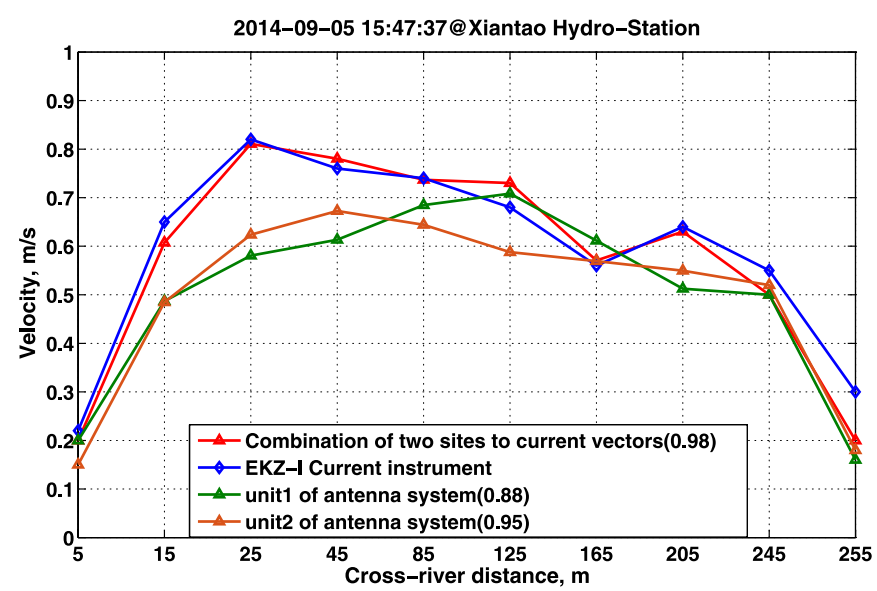

(a) Comparison between radial current, current vectors of radar system and $E K Z-I$ instrument

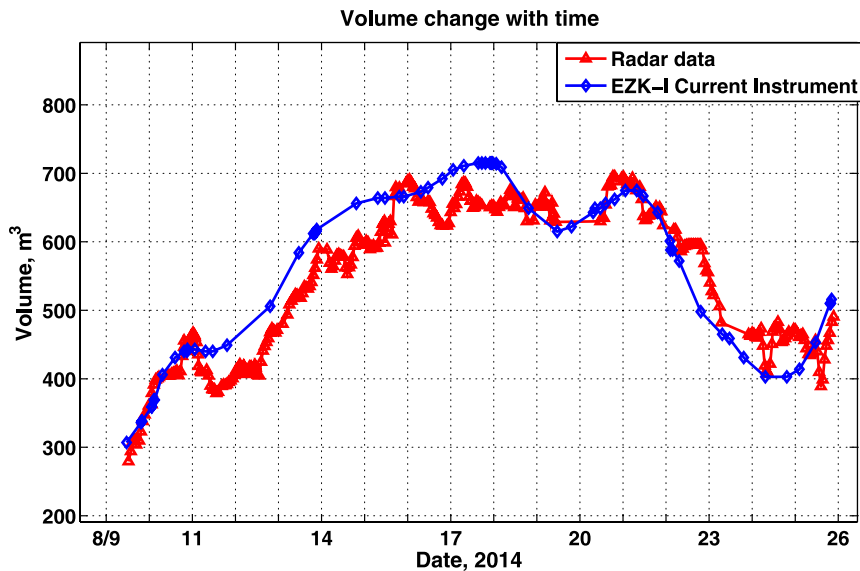

(b) River discharge with time

Fig. 8. Comparison between radar system and EKZ-I measurement

\section{Conclusion}

In this paper, a novel UHF radar system for river monitoring is presented. As compared with the traditional IF radar system, this novel radar adopts the radio frequency band-pass sampling and orthogonal demodulation for echo processing, realizes the river mapping with two-dimensional current vectors in one radar system, and the transmitting power is less than $1 \mathrm{~W}$. According to the results mentioned above, this novel radar system works well and achieves a good consistency compared with EKZ-I Current Instrument in long term observation, with a correlation coefficient more than 0.95 of the mean.

\section{Acknowledgments}

This research was supported by the National Natural Science Foundation of China (Grant No. 61072086 and No. 61001186), the Public Science and Technology Research Funds Projects of Ocean (201205032). The authors thank Xiantao Hydrological Station of the Ministry of Water Resources for providing the current data. We also wish to express our gratitude to the editor and the anonymous reviewers. 